\title{
ÜBERSICHT
}

\section{Das überarbeitete Konzept der Persönlichkeitsstörungen nach ICD-11: Neuerungen und mögliche Konsequenzen für die forensisch-psychiatrische Tätigkeit}

\author{
Nicole C. Hauser ${ }^{1}$. Sabine C. Herpertz ${ }^{2}$. Elmar Habermeyer ${ }^{1}$ \\ Eingegangen: 29. November 2020 / Angenommen: 15. Dezember 2020 / Online publiziert: 8. Januar 2021 \\ ○ Der/die Autor(en) 2021
}

\section{Zusammenfassung}

Mit der Einführung der ICD-11 (International Statistical Classification of Diseases and Related Health Problems, 11. Auflage) wird die Diagnostik von Persönlichkeitsstörungen grundlegend verändert. Die Notwendigkeit einer Abkehr von der traditionellen typologischen Auffassung und Beschreibung von Persönlichkeitsstörungen wurde aufgrund folgender Problemstellungen gesehen: Das kategoriale Konzept einer Persönlichkeitsstörung nach ICD-10 (International Statistical Classification of Diseases and Related Health Problems, 10. Auflage) begünstige die Stigmatisierung Betroffener, was dazu beitrage, dass die Diagnose in der klinischen Praxis zu selten vergeben werde. Wenn sie gestellt werde, komme es zu einer (zu) hohen Prävalenz der Persönlichkeitsstörung ,nicht näher bezeichnet“ bzw. zu hohen Komorbiditäten zwischen verschiedenen Persönlichkeitsstörungen, was Zweifel an der Reliabilität der Persönlichkeitsstörungen begründe. Außerdem sei das in der ICD-10 geforderte Kriterium der Zeitstabilität unter Berücksichtigung von Verlaufsstudien nicht mehr sinnvoll anwendbar. Der Artikel skizziert die Hintergründe für die Überarbeitung der Konzeption von Persönlichkeitsstörungen, um nachfolgend das aktuelle diagnostische Verfahren nach ICD-11 zu illustrieren. Abschließend werden die Implikationen der neuen diagnostischen Vorgaben für die forensisch-psychiatrische Schuldfähigkeitsbegutachtung diskutiert und anhand von Persönlichkeitsprofilen beispielhaft die Auswirkungen der Neukonzeption für die Therapieplanung bzw. -prognose dargestellt und diskutiert.

Schlüsselwörter Persönlichkeit · Klassifikationsysteme · Begutachtung · Therapieplanung

Prof. Dr. Elmar Habermeyer

elmar.habermeyer@puk.zh.ch

1 Klinik für Forensische Psychiatrie, Psychiatrische Universitätsklinik Zürich, Lenggstrasse 31, 8032 Zürich, Schweiz

2 Klinik für Allgemeine Psychiatrie, Universitätsklinikum Heidelberg, Heidelberg, Deutschland 


\title{
The revised concept of personality disorders according to ICD-11: innovations and possible implications for forensic psychiatric practice
}

\begin{abstract}
The introduction of the ICD-11 (International Statistical Classification of Diseases and Related Health Problems, 11th ed.) fundamentally changes the diagnostics of personality disorders. The following problems have resulted in the need to depart from the traditional typological notion of personality disorders: the categorical concept of personality disorders as defined by the ICD-10 (International Statistical Classification of Diseases and Related Health Problems, 10th ed.) favors the stigmatization of those affected. This has contributed to an underuse of the diagnosis in clinical practice. In turn, when the diagnosis is assigned the prevalence of the personality disorder "not specified" is (too) high or there are high comorbidities between different personality disorders. This casts doubt on the reliability of personality disorders. In addition, follow-up treatment studies with promising results have challenged the justification of the time stability as required by the ICD-10. This article outlines the background for the revision of the conception of personality disorders in order to illustrate the current diagnostic procedure according to the ICD-11. The new conceptions on therapy planning and prognosis are then presented and the implications for forensic psychiatric assessment of criminal liability are discussed.
\end{abstract}

Keywords Personality · Classification system · Forensic psychiatric examination · Therapy/treatment planning

\section{Kategoriale und dimensionale Modelle der Persönlichkeit/Persönlichkeitsstörung}

Die nachfolgend näher zu erläuternde Überarbeitung des Konzepts der Persönlichkeitsstörungen in der 11. Auflage der International Statistical Classification of Diseases and Related Health Problems (ICD-11) der Weltgesundheitsorganisation (WHO 2020), die im Mai 2019 verabschiedet wurde und voraussichtlich am 01.01.2022 in Kraft treten wird, fußt auf einer fachlichen Diskussion, die spätestens mit der von Kurt Schneider in den 20er-Jahren des letzten Jahrhunderts skizzierten Typologie der damals als Psychopathien beschriebenen Persönlichkeitsstörungen begonnen hat (Schneider 1923). Schneider ging es darum, auffällige Persönlichkeitsmerkmale und resultierende psychosoziale Beeinträchtigungen nicht nur aus der gesellschaftlichen Perspektive, sondern möglichst wertfrei zu betrachten. Er prägte damit einen deskriptiven Ansatz. Dennoch blieben kulturell erwartbare und akzeptierte Vorgaben - somit also auch gesellschaftliche Normen weiterhin bedeutsam, wenn nicht sogar ausschlaggebend für die Beschreibung der Persönlichkeitsstörungen. Entsprechend wurden in der 10. Version der International Statistical Classification of Diseases and Related Health Problems, der ICD-10 der World Health Organization (WHO 2004) deutlich von den Normen abweichende Verhaltensmuster und Erfahrungen Betroffener mit erheblichen negativen Konsequenzen auf sie selbst, ihre soziale Umwelt oder auf beide Bereiche als Voraussetzung für die Diagnose einer Persönlichkeitsstörung definiert. Diese erhält dadurch zwangsläufig auch eine gesellschaftliche Prägung, was insbesondere für die forensisch-psychiatrisch relevante Diagnose der dissozialen Persönlichkeitsstörung zu kritischen Anmerkungen führte. So beschrieb der Jurist Pollhähne sie als normative Diagnose (2011).
Dass sich Persönlichkeitsdiagnosen in der klinischen Praxis an der Diagnosestellung in der allgemeinen Medizin (Sass 1987) orientieren, geht wiederum mit der arbiträren Festlegung von Schwellen-Scores einher, die zwischen normal und pathologisch unterscheiden. Beispielsweise ermöglicht das Vorliegen dreier Symptomkriterien einer dissozialen Persönlichkeitsstörung nach den Vorgaben der ICD-10-Forschungskriterien (WHO 2004) die Unterscheidung zwischen normal und gestört. Dies wurde aus mehreren Gründen zunehmend als problematisch erachtet:

So sind z.B. für die Diagnose einer dissozialen Persönlichkeitsstörung 3 Merkmale vonnöten, was bei insgesamt 6 möglichen Merkmalen unterschiedlichste Kombinationen zulässt, die nicht oder kaum mehr deckungsgleich sind (Stieglitz und Freyberger 2018). Die Erfassung einer Persönlichkeitsstörung mittels der Kriterienlisten der ICD10 kann also dazu führen, dass eine Person mit der Diagnose einer bestimmten Persönlichkeitsstörung ein deutlich anderes Symptomenbild zeigen kann als eine andere Person mit derselben Diagnose. Solche, auf der Symptomebene uneinheitlichen Störungsbilder bei identischer Diagnose erschweren die Therapieplanung. In diesem Zusammenhang wurde als weiterer Kritikpunkt angeführt, dass die in kategorialen Klassifikationssystemen verwendeten Kriterienlisten aufgrund fehlender Gewichtung einzelner oder kombinierter Symptome keine Aussage über die Relevanz zutreffender Kriterien im Hinblick auf therapeutische Interventionen zulassen (Stieglitz und Freyberger 2018). Außerdem ist die hohe Komorbidität zwischen den einzelnen Persönlichkeitsstörungen zu erwähnen, der klinisch-therapeutisch kaum Rechnung getragen werden kann (Regier et al. 2013; Anderson et al. 2014). Weiterhin nachteilig für die Therapieplanung ist die gehäufte Vergabe der Diagnose „Persönlichkeitsstörung, nicht näher bezeichnet“. Als Restkatego- 
rie in der ICD-10 aufgeführt, bietet gerade diese Diagnose wenig Informationen über die individuelle Problematik der diagnostizierten Person und insbesondere keine Anhaltspunkte für eine auf die individuellen Symptome ausgerichtete Therapie (Herpertz 2018a; Skodol et al. 2011).

Auf ein anders gelagertes, aber zweifelsohne bedeutsames Problem des Konzepts der Persönlichkeitsstörung in der ICD-10 verweisen diverse Untersuchungen, die einen variablen Langzeitverlauf dieser Störungsbilder belegen konnten (Grilo et al. 2004; Zanarini et al. 2010; Gunderson 2011). Mit einer Remissionsrate bis zu $90 \%$ innerhalb von 10 Jahren weisen dabei insbesondere Borderline-Persönlichkeitsstörungen auf symptomatischer Ebene einen beachtlichen positiven Verlauf auf (Zanarini et al. 2010), während allgemein bei Persönlichkeitsstörungen eine Remissionsrate von 40-60\% innerhalb von 2 Jahren festgestellt wird (Grilo et al. 2004). Die empirische Evidenz für einen diskontinuierlichen bzw. hinsichtlich Symptomebene schwankenden Verlauf von Persönlichkeitsstörungen steht im Widerspruch zur Auffassung der ICD-10, dass sich Persönlichkeitsstörungen früh manifestieren und über die Lebensdauer stabil bleiben.

Vor diesem Hintergrund gewannen ab den 1980er-Jahren zunehmend dimensionale Modelle an Bedeutung, die Persönlichkeitseigenschaften als zeitstabile „traits“ erfassen und diese je nach Ausprägungsgrad auf Dimensionen positionieren und faktoranalytisch in unterschiedlichen Modellen zu Persönlichkeitsmustern subsumieren. Auf diese Weise gelingt es, einzelne Persönlichkeitseigenschaften in übergeordnete Persönlichkeitsfaktoren zu gruppieren. Beispielhaft kann hier auf Eysencks (1983) Dimensionen von Extraversion, Neurotizismus und Psychotizismus oder auf die Big Five (Offenheit, Gewissenhaftigkeit, Extraversion, Verträglichkeit und Neurotizismus) von Costa und McCrae (1992) verwiesen werden. Demgegenüber können sich die ICD-10-Symptomkriterien für Persönlichkeitsstörungen nicht auf entsprechende Befunde stützen (Westen und Shedler 1999).

Die vorgenannten Aspekte führten auch im forensischpsychiatrischen Kontext zu Diskussionen über die Reliabilität und Validität der Persönlichkeitsstörungsdiagnosen und ebenso bezüglich ihrer Wertigkeit für die Therapieplanung (Borchard und Gerth 2020; Habermeyer et al. 2020). Daher ist es prinzipiell zu begrüßen, dass, ausgehend von der höheren Reliabilität und Validität dimensionaler Ansätze bei der Konzeptualisierung von Persönlichkeitsstörungen und der empirischen Evidenz für fehlende qualitative Unterschiede zwischen normaler und pathologischer Persönlichkeit (Crawford et al. 2011; Hengartner et al. 2014; Fiedler und Herpertz 2016), anstelle der bisherig kategorialen Klassifikation von spezifischen Persönlichkeitsstörungen in der ICD-11 (WHO 2020) nunmehr eine dimensionale Einteilung vorgelegt wird: Dabei werden zunächst Funktionsbe- einträchtigungen geprüft, die dann anhand von 3 Schweregraden (leicht, mäßig, schwer) eingeschätzt werden. Abschließend besteht die Möglichkeit, 5 Persönlichkeitsmerkmale (negative Affektivität, Distanziertheit, Dissozialität, Enthemmung, Zwanghaftigkeit) und optional auch das Vorliegen einer Borderline-Persönlichkeit zu erfassen. Die Vorgaben der ICD-11 erlauben somit auch die Erstellung eines Profils von zeitstabileren Persönlichkeitsmerkmalen als Traits. Diese werden jedoch im Vergleich zu den in den ersten beiden diagnostischen Schritten adressierten Funktionseinbußen lediglich hinsichtlich ihres Vorhandenseins erfasst und nicht anhand ihres Schweregrades charakterisiert. Nachfolgend wird das ab 2022 zu praktizierende diagnostische Vorgehen genauer erläutert.

\section{Diagnostische Vorgaben der ICD-11}

Zur diagnostischen Abklärung einer Persönlichkeitsstörung werden zukünftig 1. diagnostisch relevante Funktionsbeeinträchtigungen geprüft und 2. das Ausmaß dieser Beeinträchtigungen bestimmt. Danach werden diese 3. Persönlichkeitsmerkmalen zugeordnet. Während also in der ICD10 zwischen 8 verschiedenen Persönlichkeitsstörungen differenziert wurde, die sich zudem kombinieren ließen, sieht die ICD-11-Klassifikation lediglich den übergeordneten Begriff „Persönlichkeitsstörung“ vor, der anhand bestimmter Leistungseinbußen, deren Schweregrad und 5 Persönlichkeitsmerkmalen charakterisiert werden kann.

\section{Relevante Funktionsbeeinträchtigungen}

Für die Diagnose einer Persönlichkeitsstörung sind Funktionsbeeinträchtigungen im Bereich des Selbst bedeutsam, die beispielsweise in Form eines instabilen und inkohärenten Identitätsgefühls und Selbstbilds oder auch eines unzulänglichen Selbstwertgefühls mit mangelnder Kompetenz, eigene Stärken und Schwächen zu erkennen, in Erscheinung treten. Eine unzulängliche Selbsteinschätzung kann mit fehlender Selbststeuerung im Zusammenhang stehen, wobei Ziele und eigene Lebenspläne nur mühevoll verfolgt bzw. umgesetzt werden können. In interpersonellen Beziehungen können Schwierigkeiten auftreten, die Perspektive anderer zu übernehmen sowie ein grundsätzliches Interesse an anderen Personen aufzubringen und aufrechtzuerhalten. Dies geht einher mit mangelnden Konfliktbewältigungsstrategien, was wiederum befriedigende Beziehungen erschwert.

Die Beeinträchtigungen zeigen sich im Bereich Kognition (z.B. dysfunktionale Entscheidungsfähigkeit), in der emotionalen Erfahrung (z.B. zu hohe oder geringe emotionale Reagibilität), im emotionalen Ausdruck und in maladaptivem Verhalten (z.B. unangemessene Impulskontrolle) und treten übergreifend in persönlichen und sozialen 
Situationen auf. Die Verhaltensmuster sind dabei dem Entwicklungsstand nicht angemessen und können nicht primär durch kulturelle Faktoren erklärt werden. Auswirkungen dieser Funktionsbeeinträchtigungen manifestieren sich in verschiedenen Lebensbereichen, darunter dem persönlichen, familiären, sozialen und beruflichen Leben, als auch in einem erheblichen Leidensdruck der betroffenen Person.

Diese Vorgaben entsprechen weitestgehend den in der ICD-10 vorgegebenen allgemeinen Kriterien für Persönlichkeitsstörungen. Allerdings müssen diese nicht mehr im späten Kindesalter bzw. während der Adoleszenz beginnen und auch nicht mehr zeitstabil sein. Vielmehr geht es nun um Symptome, die über einen Zeitraum von mindestens 2 Jahren beobachtbar sein sollen. Der nun geforderte kürzere Zeitraum der Symptomatik senkt die Diagnoseschwelle und lässt erwarten, dass die Diagnose einer Persönlichkeitsstörung zukünftig häufiger gestellt wird. Andererseits werden durch die aktuellen Veränderungen aber auch die oftmals un- bzw. nicht ausreichend beachteten Allgemeinkriterien der ICD-10 für eine Persönlichkeitsstörung hinsichtlich ihrer diagnostischen Wertigkeit betont, was die Anzahl gestellter Diagnosen auch reduzieren könnte. Genaueres wird sich erst nach einigen Jahren der Arbeit mit den modifizierten diagnostischen Vorgaben sagen lassen.

\section{Beurteilung des Schweregrads}

Die diagnostisch relevanten Leistungseinbußen werden in einem zweiten Schritt hinsichtlich ihrer Ausprägung skaliert. Diese wird anhand der Zeitstabilität von Symptomen, der Anzahl betroffener Lebensbereiche, der Ausprägung und der Chronifizierung von Funktionsbeeinträchtigungen im Gefühls- und Gedankenerleben und in Verhaltensäußerungen gemäß dem persönlichem Leidensdruck, den Einschränkungen im psychosozialen Umfeld und anhand der Selbst- und Fremdgefährdung beurteilt. Dabei werden, je nach Ausmaß der Funktionsbeeinträchtigung und ihren Auswirkungen auf verschiedene Lebensbereiche, 3 Schweregrade unterschieden:

Als leichter Ausprägungsgrad der Persönlichkeitsstörung gilt, wenn die Funktionsstörungen in manchen, aber nicht allen Persönlichkeitsbereichen auftreten, oder wenn die Einschränkungen in manchen Kontexten nicht offensichtlich erscheinen. So könnten beispielsweise Probleme in interpersonellen Beziehungen sowie bei der Erfüllung sozialer oder beruflicher Rollenerwartungen auftreten, manche Beziehungen aber aufrechterhalten und gewisse Rollenerwartungen erfüllt werden. Eine leichtgradige Persönlichkeitsstörung ist üblicherweise nicht mit Selbstoder Fremdgefährdung verbunden. Sie kann jedoch mit erheblichen Beeinträchtigungen in persönlichen, familiären, sozialen, bildungs- oder berufsspezifischen oder anderen wichtigen Funktionsbereichen einhergehen. Dabei ist für die leichte Ausprägung der Persönlichkeitsstörung ausschlaggebend, dass diese Beeinträchtigungen entweder nur einzelne Lebensbereiche betreffen oder eine Vielzahl von Funktionsbereichen in allenfalls milder Form.

Von einer mittelgradigen Persönlichkeitsstörung wird im Zusammenhang mit Beeinträchtigungen in zahlreichen Funktionsbereichen der Persönlichkeit ausgegangen, wobei manche Bereiche weniger stark betroffen sind. Beim moderaten Ausprägungsgrad der Persönlichkeitsstörung liegen in den meisten interpersonellen Beziehungen und/oder bei der Erfüllung sozialer und beruflicher Rollenerwartungen Probleme vor. Beziehungen zeichnen sich am ehesten durch Konflikte, Vermeidung, Rückzug oder extreme Abhängigkeit aus. Diese Funktionsstörungen sind mit einer erheblichen Beeinträchtigung in persönlichen, familiären, sozialen, bildungs- oder berufsspezifischen oder anderen wichtigen Funktionsbereichen verbunden, wobei in anderen Bereichen eine gewisse Funktionalität aufrechterhalten werden kann. Die Beeinträchtigungen können temporär mit Selbst- oder Fremdgefährdung in Verbindung stehen, das klinische Bild wird dadurch jedoch nicht bestimmt.

Ein schwerer Ausprägungsgrad der Persönlichkeitsstörung liegt vor, wenn Leistungseinbußen im interpersonellen Bereich vorliegen, die mit der Erfüllung sozialer Normen und Rollenerwartungen unvereinbar sind. Die Beeinträchtigungen sollen sich auf weitgehend alle Lebensbereiche erstrecken und häufig mit erheblicher Selbst- oder Fremdgefährdung verbunden sein.

Somit entsprechen insbesondere die Vorgaben hinsichtlich des schweren Ausprägungsgrades einer Persönlichkeitsstörung, z.T. aber auch die der mittelgradigen, den Vorgaben, die in der ICD-10 an das Vorliegen einer Persönlichkeitsstörung gestellt wurden. Zusätzlich können nun aber auch leichtere Auffälligkeiten bzw. umschriebene Problembereiche diagnostisch erfasst und damit auch therapeutisch relevant werden, was ebenfalls zu einer häufigeren Diagnosestellung beitragen könnte. Dabei geht die Verwendung der Begriffe ,leicht“ bzw. „schwer“ mit dem Risiko einher, dass der erstgenannte Schweregrad eine subjektiv als belastend erlebte Situation aus der Perspektive der davon Betroffenen vermeintlich bagatellisiert, während die letztgenannte Einordnung dazu verführen kann, per se von massiven Einbußen der Leistungsfähigkeit auszugehen, die jedoch keinesfalls in jeder Situation oder Intervention und auch nicht in jeder Lebensphase vorausgesetzt werden können. Insbesondere im forensisch-psychiatrischen Kontext wäre das von Nachteil, weshalb dieser Aspekt im Abschn. „Forensisch psychiatrische Aspekte“ erneut aufgegriffen werden wird. 


\section{Persönlichkeitsmerkmale oder -muster}

Vorab soll es noch um die Möglichkeiten gehen, die Funktionsbeeinträchtigungen genauer zu charakterisieren. In Ergänzung zum Schweregrad können, basierend auf dem Fünf-Faktoren-Modell (Big Five) von Costa und McCrae (Borkenau und Ostendorf 1993), 5 Persönlichkeitsmerkmale spezifiziert und miteinander in Bezug gesetzt werden, nämlich negative Affektivität, Bindungslosigkeit/ Distanziertheit, Dissozialität, Enthemmtheit und Zwanghaftigkeit. Diese Persönlichkeitsmerkmale werden wie folgt beschrieben:

Unter negativer Affektivität wird eine Neigung zu unangemessenem, intensivem und häufigem Erleben negativer Emotionen zusammengefasst, die emotionale Labilität und schlechte Emotionsregulation, negative Einstellungen sowie ein grundsätzliches Misstrauen, geringes Selbstwertgefühl und Selbstbewusstsein einschließt.

Der zweite Merkmalsbereich Bindungslosigkeit und Distanziertheit umfasst Vermeidung und Unnahbarkeit im sozialen und im emotionalen Bereich, was sich beispielsweise in einem Mangel an emotionalen Erfahrungen oder in Beziehungsvermeidung bis hin zu sozialem Rückzug äußert.

Ein dissoziales Persönlichkeitsmuster wird beschrieben, wenn soziale Interaktionen von Selbstbezogenheit und Mangel an Empathie geprägt sind, was mit der Nichtbeachtung der Rechte anderer Menschen in Verbindung steht. Dissozialität zeigt sich zudem häufig in einer Kombination von manipulativem, feindseligem und gefühllosem Verhalten gegenüber anderen.

Die Enthemmtheit umschreibt impulsives, ablenkbares und risikofreudiges Verhalten, wobei Entscheidungen von Verantwortungslosigkeit, Rücksichtslosigkeit und einem mangelnden Planungsvermögen geprägt sind.

Von Zwanghaftigkeit wird ausgegangen, wenn der Verhaltensstil perfektionistisch und rigide ist und Entscheidungen mit einem hohen Bedürfnis nach Kontrolle und Absicherung verbunden sind. Dies kann sich beispielsweise in übertriebenem Ordnungssinn, Eigensinnigkeit und unspontanem, zögerlichem Verhalten äußern.

Als zusätzliche und optionale Kategorie in der Konzeptualisierung der Persönlichkeitsmuster wird in der ICD11 die Borderline-Persönlichkeit angeführt. Basierend auf einer Vielzahl empirischer Befunde (Ellison et al. 2018; Gunderson et al. 2011) wird sie als Persönlichkeitspathologie zusammengefasst, die einerseits Störungen des Selbstkonzepts, andererseits ein problematisches interpersonelles Beziehungsverhalten und darüber hinaus dysfunktionale Emotionsregulation einschließt (Gunderson et al. 2018). Das gestörte Selbstkonzept äußert sich beispielsweise in Form von Selbstverachtung oder in einer typischerweise als Gefühl der Leere beschriebenen Selbstentfremdung. Zwischenmenschliche Beziehungen sind vielfach geprägt von einer unsteten Nähe-Distanz-Regulation, wie etwa einem übersensiblen Umgang mit Ablehnung, der sich mitunter in ausgeprägter Vermeidung von imaginierter oder tatsächlicher Zurückweisung durch andere und hohem Misstrauen diesen gegenüber zeigt. Auch sind Schwierigkeiten, die Perspektive anderer zu übernehmen, deren Grenzen zu akzeptieren und soziale Signale i. Allg. zu interpretieren, bestimmend für die Beziehungsgestaltung. Die Gefühlswelt der Borderline-Persönlichkeit wird als affektlabil oder „hyperaroused“ bezeichnet, wobei selbstschädigendes Verhalten, Suizidversuche und reaktive Aggressivität als dysfunktionale Mechanismen zur Bewältigung innerer Anspannung angeführt werden. Das Borderline-Qualifizierungsmerkmal stimmt also weitgehend mit dem BorderlineTypus der emotional-instabilen Persönlichkeitsstörung der ICD-10 überein (WHO 2004), während die anderen Störungsbegriffe aufgegeben wurden.

\section{Forensisch psychiatrische Aspekte}

\section{Begutachtung der Schuldfähigkeit}

Für die gutachterliche Arbeit ist es hilfreich, dass der Stellenwert der allgemeinen Kriterien einer Persönlichkeitsstörung bei der Überarbeitung der diagnostischen Vorgaben betont wurde. Die fehlende Berücksichtigung dieser Grundvoraussetzungen für die Diagnose einer Persönlichkeitsstörung stellte nämlich einen häufigen Fehler bei der Begutachtung dar (Habermeyer 2004). Nach Einführung der ICD-11 wird man zukünftig bei der Diagnostik Stellung zu Selbstbild, Selbstwert, Selbsteinschätzung und der Fähigkeit, Ziele und Pläne umzusetzen bzw. in Kontakt mit anderen zu treten und Konflikte zu bewältigen, nehmen müssen. Weiterhin ist zu begrüßen, dass in der ICD-11 neben kognitiven Verzerrungen und Einschränkungen der Impulskontrolle insbesondere auch emotionale Auffälligkeiten für die Diagnosestellung zu berücksichtigen sind. Schließlich kommt der entweder hohen emotionalen Reagibilität bzw. dem Empathiemangel im forensischen Kontext eine große Bedeutung zu (Habermeyer und Herpertz 2008; Herpertz 2018b), was zu den im engeren Sinne forensisch-psychiatrischen Fragestellungen überleitet.

Dabei ist zunächst relevant, dass der differenzierte dimensionale Klassifikationsansatz der ICD-11 im Bereich der Schuldfähigkeitsbegutachtung nicht konsequent umgesetzt und praktiziert werden kann, denn hier geht es um die Beantwortung dichotomer Fragen, nämlich darum, ob 1) die Diagnose der Persönlichkeitsstörung dem Merkmal der schweren anderen seelischen Abartigkeit zugeordnet werden kann, und ob diese 2) die ansonsten vorausgesetzte Steuerungsfähigkeit erheblich vermindert hat oder nicht. 
Im ersten forensisch-psychiatrischen Arbeitsschritt bedarf es also weiterhin einer präzisierenden Einordnung hinsichtlich des Schweregrades. Zu beachten ist dabei, dass es nach den Vorgaben der ICD-11 schon um Merkmale und Entwicklungen gehen kann, die sich innerhalb eines Zeitraums von 2 Jahren gezeigt bzw. ergeben haben. Ist dies der Fall, kann die Diagnose einer Persönlichkeitsstörung auch dann gestellt werden, wenn keine Auffälligkeiten im Jugend- oder im jungen Erwachsenenalter bestanden. Dadurch wird die Diagnose einer Persönlichkeitsstörung erleichtert, weshalb nochmals dringlicher vor einem Automatismus hinsichtlich Diagnose und Eingangsmerkmal zu warnen ist.

Die Möglichkeit der Schweregradeinteilung in der ICD11 kann ferner zu dem aus hiesiger Sicht voreiligen Schluss verführen, dass nur schwere Persönlichkeitsstörungen eine Einordnung als schwere andere seelische Abartigkeit rechtfertigen (können). Dies mag für den Großteil der Fälle zutreffen, jedoch wird auch bei einer schweren Persönlichkeitsstörung stets differenziert zu diskutieren und $\mathrm{zu}$ begründen sein, warum diese als schwere andere seelische Abartigkeit bezeichnet wird oder nicht. Es bleibt also auch nach Einführung der ICD-11 dabei, dass im Kontext der Schuldfähigkeitsbegutachtung eine möglichst umfassende Darstellung der Persönlichkeit anhand der biografischen Entwicklung zwingend ist. Diese basale Aufgabe wird durch die neuen diagnostischen Vorgaben eher erleichtert, denn entsprechende Auffälligkeiten können nun anhand der vorab beschriebenen Merkmalskategorien dargestellt und in ihren Auswirkungen auf das Verhalten gewichtet werden. Dabei kann bzw. muss auch zukünftig auf biografische Kenntnisse von Reaktionsweisen unter konflikthaften Belastungen und Veränderungen infolge der natürlichen Reifungs- und Alterungsschritte sowie eingeleiteter therapeutischer Maßnahmen Bezug genommen werden.

Nach wie vor sind dafür in Anlehnung an Boetticher et al. (2005) erhebliche Auffälligkeiten der affektiven Ansprechbarkeit bzw. der Affektregulation, Einengung der Lebensführung bzw. Stereotypisierung des Verhaltens, durchgängige oder wiederholte Beeinträchtigung der Beziehungsgestaltung und psychosozialen Leistungsfähigkeit durch affektive Auffälligkeiten, Verhaltensprobleme sowie unflexible, unangepasste Denkstile sowie durchgehende Störung des Selbstwertgefühls und deutliche Schwäche von Abwehr- und Realitätsprüfungsmechanismen entscheidend. Von diesen Punkten lassen sich z.B. affektive Auffälligkeiten und Störungen des Selbstwertgefühls gut in Bereichen der negativen Emotionalität abbilden, während sich eine gestörte Affektregulation in Kombination mit eingeschränkter Impulskontrolle unter dem Gesichtspunkt Enthemmung darstellen lässt.

Eine Merkmalskombination mit negativer Emotionalität und Enthemmung könnte zusätzlich mit dem Borderline-
Qualifizierungsmerkmal versehen werden und damit, bei z.B. gegebener Dissozialität, eine Persönlichkeitsstörung charakterisieren, die durch eine bei emotionaler Belastung hohe situativ-impulsive und dann auch aggressive Reaktionsbereitschaft gekennzeichnet ist. Hier würde sich die Einordnung als schwere andere seelische Abartigkeit eher anbieten als im Fall einer Dissozialität, die nicht mit negativer Emotionalität, stattdessen aber mit Distanziertheit verbunden ist. Diese Kombination charakterisiert nämlich einen dissozialen Persönlichkeitstypus, der nicht impulsiv, sondern distanziert-emotionsarm, letztlich auch strategisch vorgeht.

Die Beispiele machen deutlich, dass es auch mittelgradige Symptomausprägungen geben kann, bei denen, eine enge Bindung zwischen Persönlichkeitsproblematik und Deliktbegehung vorausgesetzt, die Annahme einer schweren anderen seelischen Abartigkeit gerechtfertigt sein kann. $\mathrm{Zu}$ denken wäre hier z. B. an Selbstwertkrisen mit einer zeitlich umschriebenen hohen Ausprägung negativer Emotionalität und impulsiver Aggressivität bei primär gegebener psychosozialer Leistungsfähigkeit.

Betreffs den im zweiten Schritt erforderlichen Aussagen zur Steuerungsfähigkeit bei einer Persönlichkeitsstörung, die unter die rechtliche Kategorie der schweren anderen seelischen Abartigkeit eingeordnet werden kann, ist unabhängig von kategorialen oder dimensionalen Störungskonzepten entscheidend, ob die Tat überhaupt und, wenn ja, in welchem Ausmaß in Bezug zur Persönlichkeitsstörung steht. Wenn das Tatgeschehen nicht auf spezifische Problembereiche (z.B. Kränkbarkeit) und Defizite (z.B. eingeschränkte Impulskontrolle) der Persönlichkeitsstörung zurückgeführt werden kann, ist die in anderen Kontexten durchaus begründete und auch relevante Diagnose nämlich für die Schuldfähigkeit nicht von Bedeutung.

Nur wenn der Symptomcharakter der Tat bejaht werden kann, ist in einem letzten Schritt zu beurteilen, ob durch die Persönlichkeitsstörung die Steuerungsfähigkeit zum Tatzeitpunkt erheblich beeinträchtigt oder gar aufgehoben gewesen ist. Diese Frage wird von der Verwendung eines dimensionalen bzw. kategorialen Störungsmodells wenig tangiert. Auch nach Einführung der ICD-11 bleibt es dabei, dass bei Persönlichkeitsstörungen nicht per se von einer erheblich verminderten Steuerungsfähigkeit ausgegangen werden kann (Boetticher et al. 2005). Die Tathintergründe und -umstände sowie die störungsbedingten Funktionsbeeinträchtigungen im Moment der Tat sind weiterhin abzuklären. Um Aussagen betreffs der zum Tatzeitpunkt bestehenden bzw. fehlenden Freiheitsgrade erfassen zu können, bleibt die detaillierte Analyse der Ausgangsbedingungen eines Delikts, des Tatablaufs, der handlungsleitenden Motive, der Täter-Opfer-Beziehung und des Nachtatverhaltens entscheidend (Sass und Habermeyer 2007; Dressing et al. 2021). In diesem Kontext hat Kröber (2016, 2020) 
mit guten Argumenten vor einer einseitigen Orientierung an der exekutiven Steuerungsfähigkeit gewarnt, denn in Anlehnung an Janzariks Begriff der Einsichtssteuerung (2020) können nicht zuletzt bei im Beziehungskontext begangenen Straftaten eine gedankliche Einengung auf den emotional hoch besetzten Konflikt und weitere motivationale Aspekte eine verminderte Steuerungsfähigkeit bedingen. In diesem Kontext gibt das überarbeitete Konzept der Persönlichkeitsstörungen, wie vorab beispielhaft dargestellt, allerdings die Möglichkeit, entsprechende Einbußen hinsichtlich ihres Schweregrades zu erfassen und bestimmten Problembereichen zuzuordnen (z.B. der Enthemmung hinsichtlich der exekutiven oder der negativen Emotionalität bezüglich der motivationalen Steuerungsfähigkeit).

\section{Therapieplanung}

Die Behandlung der jeweiligen Persönlichkeitsstörungen richtet sich an den für die Diagnosestellung relevanten Funktionseinbußen aus. Es geht also primär um die Behandlung von Symptomen, die einen unmittelbaren Leidensdruck sowie Probleme in der sozialen Interaktion begünstigen und weniger um eine Veränderung von Persönlichkeitsmerkmalen. Dieser Ansatz ist nicht nur pragmatisch, sondern dürfte den Betroffenen auch leichter vermittelbar sein, denn er zielt unmittelbarer auf die Beeinträchtigungen $\mathrm{ab}$, die ihnen oder ihrem Umfeld Probleme bereiten.

Allerdings können auch die im letzten diagnostischen Schritt zu berücksichtigenden Persönlichkeitsfaktoren hinsichtlich ihrer klinischen Relevanz eingeordnet und damit als therapeutisch bedeutsam adressiert werden. Dies betrifft im forensisch-psychiatrischen Kontext aber vorwiegend Aussagen zur Therapieprognose, die in der Schweiz schon im Anlassverfahren gefordert sind und in Deutschland im Verlauf der Unterbringung relevant werden. Schließlich ergeben sich bei ausgeprägter negativer Emotionalität, die sich in pessimistischen und schwankenden Stimmungen äuBert, und einem dissozialen, aggressiven und enthemmten Lebensstil, der sich in häufigen Wutausbrüchen, haltlosen Äußerungen und feindseligen bis zu gewalttätigen Reaktionen auf Frustrationen zeigt, andere therapeutische Optionen als bei der Kombination von Dissozialität mit Zwanghaftigkeit und Distanziertheit, denn dieses Erscheinungsbild beschreibt ein unempathisch-manipulatives, selbstsicheres und gleichzeitig perseverierendes, perfektionistisches und planerisches Persönlichkeitsmuster. Von forensischpsychiatrischer Bedeutung ist in diesem Beispiel, dass das Vorliegen von negativer Emotionalität in Form von Ängsten und depressiven Symptomen auf einen günstigeren Therapieverlauf im Zusammenhang mit dissozialen Persönlichkeitszügen hinweisen kann als eine geringe Ausprägung negativer Affektivität, die einen geringen Leidensdruck und wenig Änderungsmotivation bei einem Patienten oder einer Patientin nahelegt (Hodgins und De Brito 2009).

Im Hinblick auf die Enthemmung kommen außerdem auch medikamentöse Behandlungsoptionen zur Reduktion der Impulsivität oder kognitiv-verhaltenstherapeutische Programme wie die Dialektisch Behaviorale Therapie für forensische Patientinnen und Patienten (DBT-F, Ivanoff und Marotta 2018) zur Förderung der Emotionsregulation in Betracht. Vor dem Hintergrund des zentralen Therapiewirkfaktors Beziehung (Horvath et al. 2011) lässt das Persönlichkeitsmerkmal der Distanziertheit und Bindungslosigkeit erwarten, dass der für einen erfolgreichen Therapieverlauf entscheidende Beziehungsaufbau schwierig wird. Unabhängig von den vorgenannten Merkmalen bzw. Merkmalsmustern ist das Vorhandensein des BorderlineMerkmals von therapeutischer Bedeutung, da hier mit der DBT und Schematherapie bewährte Behandlungsoptionen existieren.

Somit wird die Therapieplanung durch das neue Konzept der Persönlichkeitsstörungen vereinfacht bzw. individualisiert, denn die Bedingungen des Einzelfalls lassen sich differenzierter abbilden. Hier wird die dimensional ausgerichtete und damit auch komplexere Vorgehensweise in klinisch relevanter Hinsicht zum Vorteil. Wenn, wie in der Schweiz, zur Anordnung einer therapeutischen Maßnahme auch Aussagen zur Therapierbarkeit bzw. den Erfolgsaussichten einer Therapie gefordert werden, bietet die ICD11-Klassifikation die Möglichkeit, gegebene bzw. fehlende Therapieoptionen und deren Erfolgsaussichten genauer zu skizzieren, als es angesichts der variablen Verläufe und heterogenen Symptomenbilder der einzelnen ICD-10-Persönlichkeitsstörungen möglich war.

\section{Fazit}

Die kategoriale Diagnostik der Persönlichkeitsstörungen wurde aus unterschiedlichen und auch guten Gründen kritisiert. Nicht zuletzt mit den zunehmenden Erkenntnissen zur Variabilität der Symptomatik wuchs das Bedürfnis nach einer Betrachtung, die individueller, damit naturgemäß aber auch komplexer wird. In der ICD-11 wurde dies nun umgesetzt, wobei die grundlegende Veränderung auch Folgen für die forensisch-psychiatrische Begutachtung und Therapieplanung hat: Bei der erstgenannten Aufgabe muss aufgrund der Senkung der Diagnoseschwelle nochmals stärker ein Augenmerk auf die Unterscheidung zwischen klinischer Diagnose und dem juristischen Eingangskriterium der schweren anderen seelischen Abartigkeit gelegt werden. Hinsichtlich Therapieplanung und auch Festlegungen zur Therapieprognose ergeben sich durch die detaillierten Unterscheidungsmöglichkeiten neue und aus hiesiger Sicht vielversprechende Möglichkeiten, den Gegebenheiten des 
Einzelfalls bei Aussagen zu Therapiemöglichkeiten, aber auch -prognose Rechnung zu tragen.

Funding Open access funding provided by University of Zurich.

Interessenkonflikt S.C. Herpertz ist Mitglied der WHO (World Health Organization) Task Group zur Klassifikation von Persönlichkeitsstörungen. N.C. Hauser und E. Habermeyer geben an, dass kein Interessenkonflikt besteht.

Open Access Dieser Artikel wird unter der Creative Commons Namensnennung 4.0 International Lizenz veröffentlicht, welche die Nutzung, Vervielfältigung, Bearbeitung, Verbreitung und Wiedergabe in jeglichem Medium und Format erlaubt, sofern Sie den/die ursprünglichen Autor(en) und die Quelle ordnungsgemäß nennen, einen Link zur Creative Commons Lizenz beifügen und angeben, ob Änderungen vorgenommen wurden.

Die in diesem Artikel enthaltenen Bilder und sonstiges Drittmaterial unterliegen ebenfalls der genannten Creative Commons Lizenz, sofern sich aus der Abbildungslegende nichts anderes ergibt. Sofern das betreffende Material nicht unter der genannten Creative Commons Lizenz steht und die betreffende Handlung nicht nach gesetzlichen Vorschriften erlaubt ist, ist für die oben aufgeführten Weiterverwendungen des Materials die Einwilligung des jeweiligen Rechteinhabers einzuholen.

Weitere Details zur Lizenz entnehmen Sie bitte der Lizenzinformation auf http://creativecommons.org/licenses/by/4.0/deed.de.

\section{Literatur}

Anderson J, Snider S, Sellbom M, Krueger R, Hopwood C (2014) A comparison of the DSM-5 section II and section III personality disorder structures. Psychiatry Res 216(3):363-372. https://doi. org/10.1016/j.psychres.2014.01.007

Boetticher A, Nedopil N, Bosinski H, Sass H (2005) Mindestanforderungen für Schuldfähigkeitsgutachten. Nervenarzt 76:1154-1160

Borchard B, Gerth J (2020) Die Relevanz eines kohärenten forensischen Beurteilungs- und Behandlungsprozesses. Forens Psychiatr Psychol Kriminol 14(2):197-211. https://doi.org/10.1007/ s11757-020-00586-0

Borkenau P, Ostendorf F (1993) NEO-Fünf-Faktoren Inventar (NEOFFI) nach Costa und McCrae. Hogrefe, Göttingen https://doi.org/ 10.1026//0084-5345.28.2.145

Costa P, McCrae R (1992) Four ways five factors are basic. Pers Individ Dif 13(6):653-665. https://doi.org/10.1016/0191-8869(92)90236I

Crawford MJ, Koldobsky N, Mulder R, Tyrer P (2011) Classifying personality disorder according to severity. J Personal Disord 25(3):321-330. https://doi.org/10.1521/pedi.2011.25.3.321

Dressing H, Mokros A, Habermeyer E (2021) Persönlichkeitsstörungen. In: Dressing H, Habermeyer E (Hrsg) Psychiatrische Begutachtung, 7. Aufl. Elsevier, München, S 319-335

Ellison WD, Rosenstein LK, Morgan TA, Zimmerman M (2018) Community and clinical epidemiology of borderline personality disorder. Psychiatr Clin North Am 41(4):561-573. https://doi.org/10. 1016/j.psc.2018.07.008

Eysenck HJ (1983) Psychophysiology and personality: extraversion, neuroticism and psychoticism. Individ Differ Psychopathol. https://doi.org/10.1016/B978-0-12-273903-3.50007-9

Fiedler P, Herpertz SC (2016) Persönlichkeitsstörungen, 7. Aufl. Beltz, Weinheim

Grilo CM, Sanislow CA, Gunderson JG, Pagano ME, Yen S, Zanarini MC, Shea MT et al (2004) Two-year stability and change of schizotypal, borderline, avoidant, and obsessive-compulsive personality disorders. J Consult Clin Psychol 72(5):767-775. https:// doi.org/10.1037/0022-006X.72.5.767
Gunderson JG, Stout RL, McGlashan TH, Shea MT, Morey LC, Grilo CM, Zanarini MC et al (2011) Ten-year course of borderline personality disorder: psychopathology and function from the collaborative longitudinal personality disorders study. Arch Gen Psychiatry 68(8):827-837. https://doi.org/10.1001/ archgenpsychiatry.2011.37

Gunderson JG, Herpertz SC, Skodol AE, Torgersen S, Zanarini MC (2018) Borderline personality disorder. Nat Rev Dis Primers 4(1):18029. https://doi.org/10.1038/nrdp.2018.29

Habermeyer E (2004) Typische Fallstricke bei der Begutachtung von Persönlichkeitsstörungen. PTT Persönlichkeitsstörungen Theor Ther 8:85-92

Habermeyer E, Herpertz SC (2008) Emotionalität und Schuldfähigkeit. Psychiatrie 5:172-181

Habermeyer E, Mokros A, Briken P (2020) Die Relevanz eines kohärenten forensischen Beurteilungs-und Behandlungsprozesses: Großer Wurf oder alter Wein in undichtem Schlauch? Forens Psychiatr Psychol Kriminol 14(2):212-219. https://doi.org/10. 1007/s11757-020-00592-2

Hengartner MP, Ajdacic-Gross V, Rodgers S, Müller M, Rössler W (2014) The joint structure of normal and pathological personality: further evidence for a dimensional model. Compr Psychiatry 55(3):667-674. https://doi.org/10.1016/j.comppsych.2013.10. 011

Herpertz SC (2018a) Neue Wege der Klassifikation von Persönlichkeitsstörungen in ICD-11. Fortschr Neurol Psychiatr 86:150-155

Herpertz SC (2018b) Empathie und Persönlichkeitsstörungen aus neurobiologischer Sicht. Forens Psychiatr Psychol Kriminol 12(3):192-198. https://doi.org/10.1007/s11757-018-0480-5

Hodgins S, De Brito SA (2009) Die Antisoziale Persönlichkeitsstörung des DSM-IV-TR - Befunde, Untergruppen und Unterschiede zu Psychopathy. Forensische Psychiatrie, Psychologie, Kriminologie 3:116-128

Horvath AO, Del Re AC, Flückiger C, Symonds D (2011) Alliance in individual psychotherapy. Psychotherapy 48:9-16. https://doi. org/10.1037/a0022186

Ivanoff A, Marotta PL (2018) DBT in Forensic Settings. In: Swales MA (Hrsg) The Oxford Handbook of Dialectical Behaviour Therapy. https://doi.org/10.1093/oxfordhb/9780198758723.013. 14

Janzarik W (2020) Grundlagen der Einsicht und das Verhältnis von Einsicht und Steuerung. Forens Psychiatr Psychol Kriminol 10:406-411

Kröber H-L (2016) Die Beurteilung der Steuerungsfähigkeit bei psychischen Störungen. Forens Psychiatr Psychol Kriminol 10:181-188

Kröber H-L (2020) Konzepte und Implikationen der verminderten Schuldfähigkeit. Forens Psychiatr Psychol Kriminol 10:381-392

Pollähne H (2011) Kriminalprognostik. Untersuchungen im Spannungsfeld zwischen Sicherheitsrecht und Rechtssicherheit

Regier DA, Narrow WE, Clarke DE, Kraemer HC, Kuramoto SJ, Kuhl EA, Kupfer DJ (2013) DSM-5 field trials in the United States and Canada, part II: test-retest reliability of selected categorical diagnoses. Am J Psychiatry 170(1):59-70. https://doi.org/ 10.1176/appi.ajp.2012.12070999

Saß H (1987) Psychopathie-Soziopathie-Dissozialität: Zur Differentialtypologie der Persönlichkeitsstörungen. Springer, Berlin

$\mathrm{Sa}$ H, Habermeyer E (2007) Die Begutachtung von Persönlichkeitsstörungen aus psychopathologischer Sicht. Forens Psychiatr Psychol Kriminol 1:156-161

Schneider K (1923) Die psychopathischen Persönlichkeiten, 1. Aufl. Deuticke, Wien

Skodol AE, Bender DS, Morey LC, Clark LA, Oldham JM, Alarcon RD, Krueger RF, Verheul R, Bell CC, Siever LJ (2011) Personality disorder types proposed for DSM-5. J Personal Disord 25(2):136-169. https://doi.org/10.1521/pedi.2011.25.2.136 
Stieglitz RD, Freyberger HJ (2018) Diagnostik von Persönlichkeitsstörungen in ICD-10/-11 und DSM-5. Z Psychiatr Psychol Psychother 66(2):71-83. https://doi.org/10.1024/1661-4747/a000343

Westen D, Shedler J (1999) Revising and assessing axis II, part I: developing a clinically and empirically valid assessment method. Am J Psychiatry 156(2):258-272. https://doi.org/10.1176/ajp. 156.2.258

World Health Organization (2004) International statistical classification of diseases and related health problems, 10. Aufl. WHO, Genf
World Health Organization (2020) International statistical classification of diseases and related health problems, 11. Aufl. (https://icd. who.int/)

Zanarini MC, Frankenburg FR, Reich DB, Fitzmaurice G (2010) Time to attainment of recovery from borderline personality disorder and stability of recovery: a 10-year prospective follow-up study. Am J Psychiatry 167(6):663-667. https://doi.org/10.1176/appi. ajp.2009.09081130 